Articles

\title{
CommunitySensor: towards a participatory community network mapping methodology
}

Aldo de Moor
CommunitySense, The Netherlands

Corresponding Author.

ademoor@communitysense.nl

Participatory community network mapping can support collaborative sensemaking within and across communities and their surrounding stakeholder networks. We introduce the CommunitySensor methodology under construction. After summarizing earlier work, we show how the methodology uses a cyclical approach by adopting a Community Network Development Cycle that embeds a Community Network Sensemaking Cycle. We list some observations from practice about using community network mapping for making inter-communal sense. We discuss how extending the methodology with a patterndriven approach benefits the building of bridges across networked communities, as well as the sharing of generalized lessons learnt. To this purpose, a community collaboration pattern language is essential. We show initial work in developing and using such a language by examining the cross-case evolution of core community network interaction patterns.

\section{Introduction}

Society faces many wicked problems, such as climate change, financial and economic crises, terrorism, and wars. Wicked problems cannot be solved by organizations or communities on their own, but instead require a "movement of movements" in order to find solutions that scale and are sustainable (Klein, 2015). Achieving the required collective impact means getting the commitment of relevant actors from across different

de Moor, A. (2017). CommunitySensor: towards a participatory community network mapping methodology. The Journal of Community Informatics, 13(2), 35-58.

Date submitted: 2017-03-28. Date accepted: 2017-08-21.

Copyright (C), 2017 (the author as stated). Licensed under the Creative Commons AttributionNonCommercial-ShareAlike 2.5. Available at: www.ci-journal.net/index.php/ciej/article/view/1382 
sectors to realize a common agenda, while working towards shared measurement, mutually reinforcing activities, continuous communication, and backbone support (Kania et al, 2014).

One way to achieve the required coordination is to develop official "backbone organizations" that align efforts of various initiatives (Irby \& Boyle, 2014). However, such a resource-heavy approach is often not feasible. Moreover, finding solutions to wicked problems is complicated by their inherent social complexity. Addressing the fragmenting force of wicked problems therefore calls for a process of collaborative sensemaking using new understandings, processes, and tools in which stakeholders across the board collaborate in complex thinking and decision-making processes (Conklin, 2006).

Communities are the building blocks of collaboration in today's networked world. They consist of people from often different organizations and backgrounds working together for mutual benefit, in the process developing strong relations, and weaving a web of vibrant interactions (de Moor, 2015). Communities of practice, communities of interest, innovation communities, and so on, help to bridge knowledge gaps and cross collaboration barriers within and between organizations. However, achieving collective impact at scale goes beyond the individual community, and involves aligning resources, practices, and initiatives of multiple communities in a subtle process of inter-communal sensemaking, which we called knowledge weaving (de Moor, 2015).

Participatory community mapping - visualizing and jointly with community members making sense of the collaboration ecosystem of communities - is key to making communities work (together) better. Earlier, we presented initial work on a participatory community mapping methodology and showed how it was instrumental in helping the Tilburg urban farming community make sense of itself (de Moor, 2015b). In the current paper, we deepen our exploration of the emerging methodology.

Communities can be defined as sets of relationships where people interact socially for mutual benefit (Andrews, 2002). It is important to realize that communities and (social) networks are not two completely different organizational forms. Instead, they are part of a continuum. The network aspects refer to the relationships, personal interactions, and connections among participants, providing affordances for learning and collaboration; the community aspect refers to the development of a shared identity around a topic or set of challenges (Wenger et al., 2011). We might therefore say that communities are "densifications" in a rich social network substrate. To be more precise about the scope of our mapping efforts, we therefore redefine our methodology as a participatory community network mapping methodology. This does not mean we try to change the whole outside world context of the collaborative community being supported. Rather, we acknowledge that the boundaries of such a community are fuzzier than they may seem at first sight. For successful community development, at least a basic representation and understanding of this larger network context is a necessary condition. 
In this article, we first introduce what we mean by participatory community network mapping for collaborative sensemaking and summarize the current state of our methodology, which we call CommunitySensor.

\section{Introducing CommunitySensor}

We start by examining the need for a methodology for participatory community network mapping. We then summarize earlier methodology development work that focused on supporting intra-communal sensemaking. We outline how this initial approach has evolved into a cyclical approach for community network development and sensemaking. We illustrate that approach through a case we did on performing a participatory "force field analysis" of a collaboration ecosystem around a specific theme of investigation.

\section{The need for a participatory community network mapping methodology}

To improve their collaboration, community members and network stakeholders need to continually make sense of it (de Moor, 2015). This collaborative sensemaking process involves developing a common process of reaching a shared understanding about the collaboration, including the various interests and perspectives of the community members and surrounding stakeholder networks. Collaborative sensemaking helps community members jointly find out what their collaboration is about, what relationships and interactions their community and its context consist of, what collaboration resources are available, and what concrete opportunities exist for better working communities.

Sensemaking is the process by which people give meaning to experience. Sensemaking is much more, however, than just a random process of reflection. Weick defined the following essential properties: sensemaking is grounded in identity construction, is retrospective, is about actively acting upon and creating the environment, is a social and ongoing process, revolves around extracting cues to help make sense, and is not so much about accuracy but plausibility (Weick, 1995).

Participatory community network mapping is a core communal sensemaking activity. We define it as the participatory process of capturing, visualizing, and analyzing community network relationships and interactions and applying the resulting insights for community sensemaking, building, and evaluation purposes. There are many variations of (more or less participatory) community mapping, including geographical community-mapping, e.g. tribal communities mapping their local rainforest resources ( http://www.mappingforrights.org); concept mapping to visualize the context of common concepts from the lens of a focus question (Novak \& Cañas, 2008), dialogue mapping to capture the issues, positions and arguments in meeting discussions (Conklin, 2006), and social network mapping, in which the structural properties of social networks are analyzed, for example to detect emerging community roles (Smith, 2014).

Although the community mapping approaches mentioned all have their merits, they are insufficient from the point of view of (1) supporting sustained and scalable participatory community network cultivation along the lines of (Wenger et al, 2011). Moreover, our 
methodology aims to (2) integrate insights from the emerging field of knowledge cartography: how to improve our capacity to create and use high-level meaningful (digital) knowledge visualizations (Selvin \& Shum, 2014), thus leveraging the sensemaking capacity needed for collective intelligence and impact.

A methodology includes a description of the process to be performed and of the roles involved in the process, assigns responsibilities to activities and people and gives recommendations in form of best practices and guidelines (IEEE Computer Society, 1990 in Simperl \& Luczak-Rösch, 2014). We first summarize earlier work on our methodology, then outline the current state of what has grown into the CommunitySensor methodology.

\section{Earlier work on the methodology: intra-communal sensemaking}

We developed an initial version of our methodology as part of a project to stimulate urban farming in the Dutch province of Noord-Brabant, focusing on an emerging community of urban farmers around the city of Tilburg. To this purpose, we developed an online map using the online network visualization tool Kumu (http://bit.ly/1L0jusT). By its very nature, such community mapping is participatory, meaning that relevant stakeholders need to be (inter)actively involved in providing and interpreting map data.

In (de Moor, 2015b), we showed how participatory community mapping requires an appropriate language (what types of elements and connections, what perspectives and layout?), tools (how particular functionalities of online tools can help in the storytelling and visualization, analysis, and sharing of maps, how physical tools like meetings and plenary meetups can help to develop ownership), and processes (e.g. how best to capture, analyze, use, and evolve maps in community practice).

Lessons learnt about map data capturing in practice include that it helps to distinguish between a - possibly external - master map maker (the architect designing the mapping language, tools, and processes, configuring and creating the initial map, and providing general support) and domain map makers from the community (who can keep adding new elements and connections to the initial map as the collaboration grows). It is also important to balance completeness and feasibility: trade-offs are needed in frequency \& granularity as only limited map making resources are generally available (e.g. making only quarterly official updates, modeling just organizational participants instead of individuals, as accurately adding the latter would make the map unmaintainable when possibly many hundreds of quickly changing persons are involved). We also observed a constructive "friendly peer pressure" arising, which led community members to contribute data since they saw others represented on the map and they wanted to be seen as well. However, it is important to avoid having participants game the system by, for example, providing data about the same activity in different formats, while suggesting they are different activities. In this way, participants might artificially inflate their perceived involvement in the community, so that they get more recognition than justified. One way to deal with this is by making the criteria for the inclusion of elements and connections explicit and grounded in the social norms of the community. 
As to interpreting and using the maps, we learnt that community members are particularly interested in map views that focus on the direct context of their own organization or activity, whereas community managers are generally more interested in getting the broader, bird's eye views of the total map for community management purposes (e.g. informing them which activities/participants act as hubs to which new activities can be linked, or becoming aware of the need to intervene when crucial activities or participants are "dangling" on the periphery of the map whereas they should play a central role).

\section{Participatory community network mapping: a cyclical aproach}

The overall purpose of the CommunitySensor methodology under construction is to help communities make better sense of and strengthen their collaboration through participatory community network mapping. This evolutionary process consists of two interconnected cycles, both driven by the mapping process, and named after the main purpose of that cycle : a Community Network Development Cycle and a Community Network Sensemaking Cycle.

\section{The Community Network Development Cycle}

At the macro-level, there is the ongoing Community Network Development Cycle, consisting of four subsequent stages: community network mapping, community network sensemaking, community network building, and community network evaluation. The development process starts by (1) mapping the community network through visualizing the most relevant pieces of the community network into map elements, connections, and views; (2) using these mapping artifacts to make sense of the collaborative common ground of the community network in terms of issues, priorities, and next actions; (3) designing and implementing community network interventions needed to carry out these actions, and so building the community and making its collaboration grow in the context of its wider network; (4) evaluating the effects of these interventions in terms of collecting stories, data and indicators to provide the inputs for the next round of mapping. This process is to be repeated continuously, resulting in ever richer and more situated maps, a deeper joint sense of awareness and ownership of the collaboration ecosystem the community network consists of, and more effective community building interventions, the results of which are measured in terms of stories, data, and indicators that ihelp nspire the next round of development (see figure 1).

By continuing to repeat this cycle, sustainability and scalability can be achieved from the bottom-up, grounded in the increasingly clear needs and capabilities of the community network. Such a bottom-up process approach is essential for social innovations to ultimately have a chance of having systemic impact (Murray and Mulgan, 2010). This is literally a spiraling development process, to which the building interventions of possibly many communities and other, external success factors and constraints may contribute. Many of these external aspects will not be under the control of the community trying to make sense of its own niche in the larger collaboration ecosystem. Hence, the crucial role of the network mapping process as a continuous attempt to better understand this rich and evolving collaborative community context. 


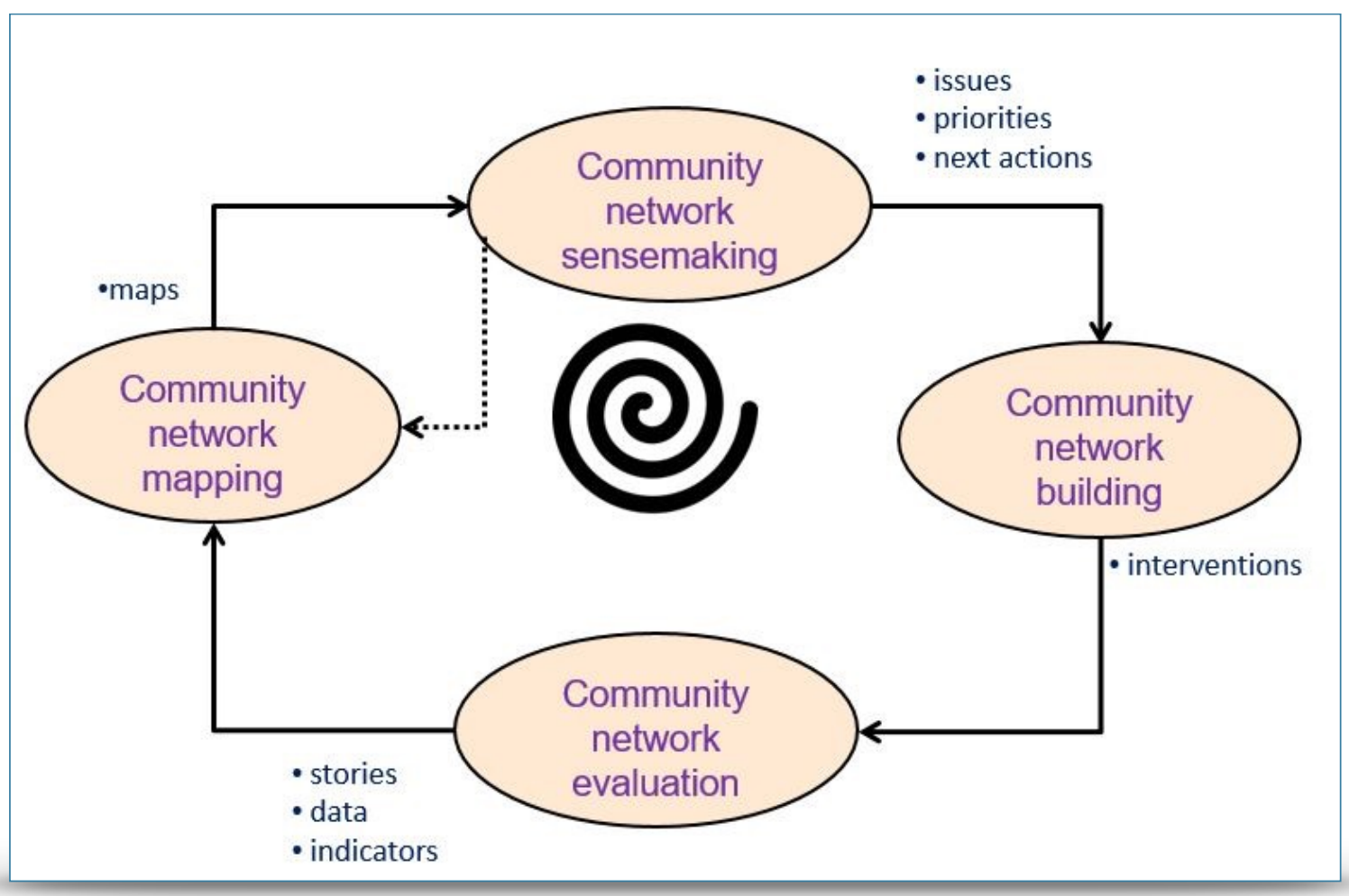

Figure 1: The Community Network Development Cycle

\section{The Community Network Sensemaking Cycle}

The linear nature of the Community Network Development Cycle is only an approximation. In reality, backtracking often occurs. For example, when designing community network building interventions, it is sometimes necessary to revisit the sensemaking stages, as there are unclarities about, for example, the commonly understood meanings of specific concepts. However, there is one specific subcycle that is so prevalent that we model it explicitly: the Community Network Sensemaking Cycle. This cycle consists of the interplay between the community network mapping and community network sensemaking-stages of the Community Network Development Cycle.

Within this sensemaking cycle, map makers first map the essence of the core community and its surrounding collaborative network of related stakeholders and resources. This initial map - which we call the "seed map" - is composed out of data from source documents, such as project reports and spreadsheets, as well as interviews with core community members. Besides being a necessary condition for kickstarting the sensemaking process, creating this initial artifact also helps to check whether the mapping language selected is sufficiently tailored to the needs of the community. We found that investing in getting this seed map "right" in terms of using the proper mapping language and having a sufficient number of community-recognizable example elements and connections is very important for sparking engaged sensemaking conversations. 


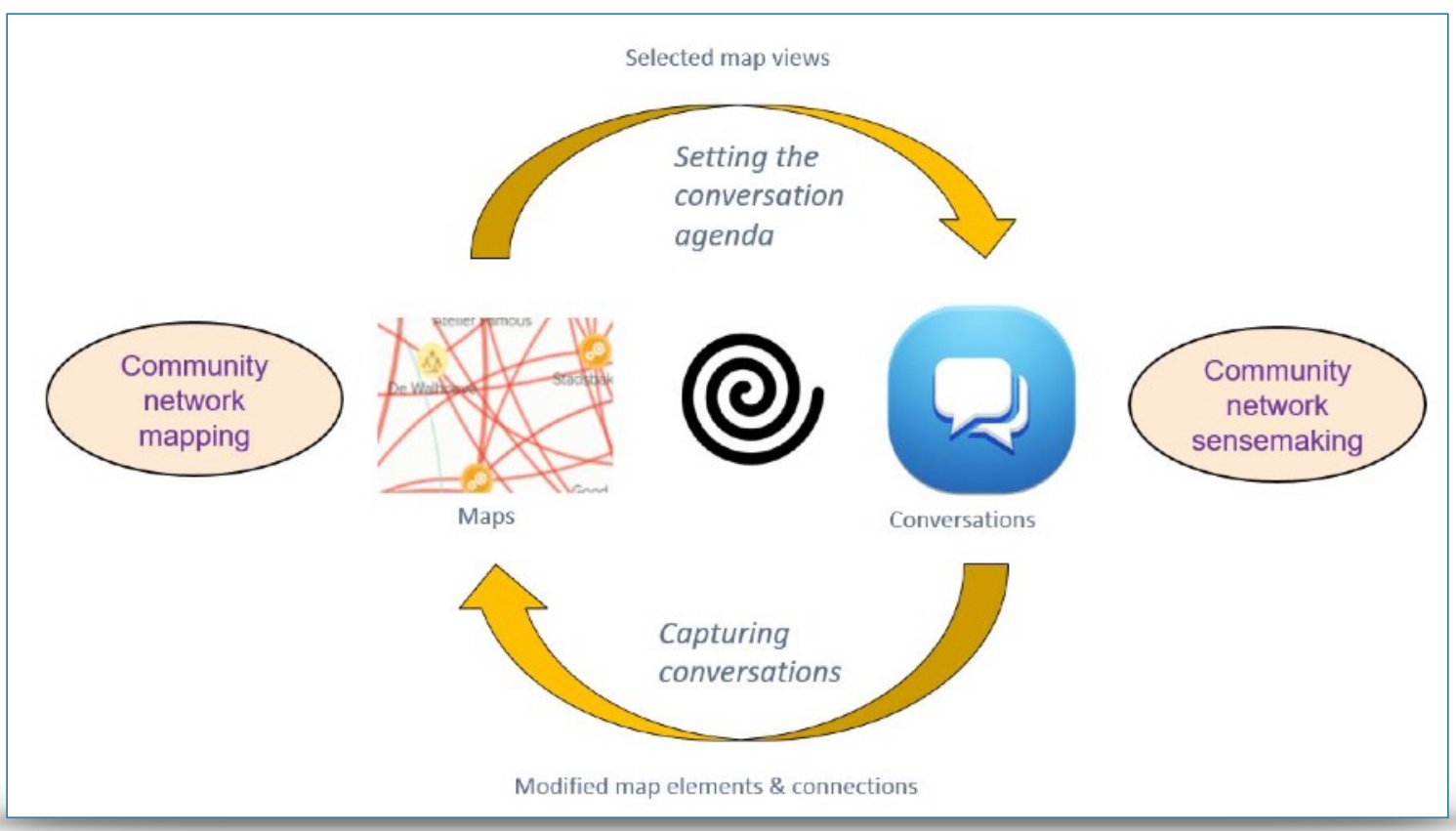

Figure 2: The Community Network Sensemaking Cycle

The seed map and subsequent map versions set the agenda for the conversations in the sensemaking process. This agenda consists of those views on the map that are likely most relevant for the subset of participants in a specific sensemaking conversation.

Outcomes of these sensemaking conversations are issues, priorities and next actions to be taken in the subsequent community network building stage. The sensemaking cycle may need to be iterated several times before enough common sense has been made and captured in the map. In each iteration, new insights from the conversations about the emerging collaboration are represented in terms of (1) elements and connections to be added or modified and (2) which of these elements and connections are to be focused on next to further the collaboration. These sensemaking conversations can take many forms and we are still actively experimenting with what conversation agenda and what type of conversation best fit the needs of the community.

\section{Applying the Community Network Sensemaking Cycle: performing a participatory "force field analysis" of a thematic collaboration ecosystem}

To illustrate how the sensemaking cycle works, we share how using it played out in a practical situation. The case concerns an organization in the public domain interested in using our participatory community network mapping methodology for doing a "force field analysis" of the collaboration ecosystem around a specific theme of investigation (combating care fraud)1, The force field analysis was to act as a precursor to an indepth data analysis that was to investigate the occurrence of that issue in practice. The effort was also to help construct bridges between two organizational departments working on that issue, but which were still too unfamiliar with each other's ways of

1 As this concerned a sensitive internal matter, no identifying details are shared here. 
working. Besides providing the focus for that investigation, as well as fostering interdepartmental collaboration, the community network map produced also provides an "action context" from which the results of that data analysis (ongoing as of the publication of this article) can be interpreted and lead to follow-up steps.

In a series of seven "working sessions", a map consisting of 285 elements and 442 connections was created. which according to the community members sufficiently reflected the essence of their local force field, including the main issues, priorities, and ideas for the next actions to be taken.

In the first session, representatives from both departments met with the master map maker and agreed upon the language (element and connection types as well as key perspectives and layouts) to be used by going through a number of typical examples. The main element types to be put on the map included Themes, Workflows, Organizations, Stakeholders, Data Sources, Cases, and Questions/Issues. In the second session, representatives from both departments jointly sketched the contours of the map. The next four sessions consisted of meetings with representatives from the two departments separately, allowing for their ideas and concerns to be collected and interpreted without running the risk of their premature interactions leading to "forced consensus".

At the final sensemaking session, representatives from both departments participated jointly again. The goal of that session was not to add more elements and connections, but to weigh the already represented map items in terms of which elements 2 should be focused on in the next action: the data analysis. To this purpose, the representatives from both departments had been asked in their respective last individual sessions to do a "quick and dirty" prioritizing of the map elements from the point of view of their own department. In the plenary session, each of these tentative departmental priorities was briefly discussed, after which a consensus decision was made on the final joint priorities. In total, 72 of the 285 elements were agreed to be a common focus for the data analysis.

Figure 3 shows an excerpt of the much larger resulting map. Interdepartmental priorities are visually indicated by a green border. Through the process, a joint sense of ownership (and thus action potential) between the two departments was achieved. It also provided the organizational context and focus for the data analysts to make their subsequent analysis as relevant as possible. However, this is not just an internal organizational community of practice in the making. Both departments are linking pins in a large community network of collaborating organizations and stakeholders, all of which have been put on the map as well (in total in this initial investigation already 39 organizations/departments and 26 types of stakeholders involved in the local care fraud ecosystem were identified). In future sessions, representatives from these actors could

2 The focus was on the elements, not the connections, as we represented all observations and insights in the elements, with the connections only providing the context of each element. This because we found that participants more naturally select elements over connections in sensemaking. 
be invited to make sense of "their" part of the map, further scaling up community network development efforts.

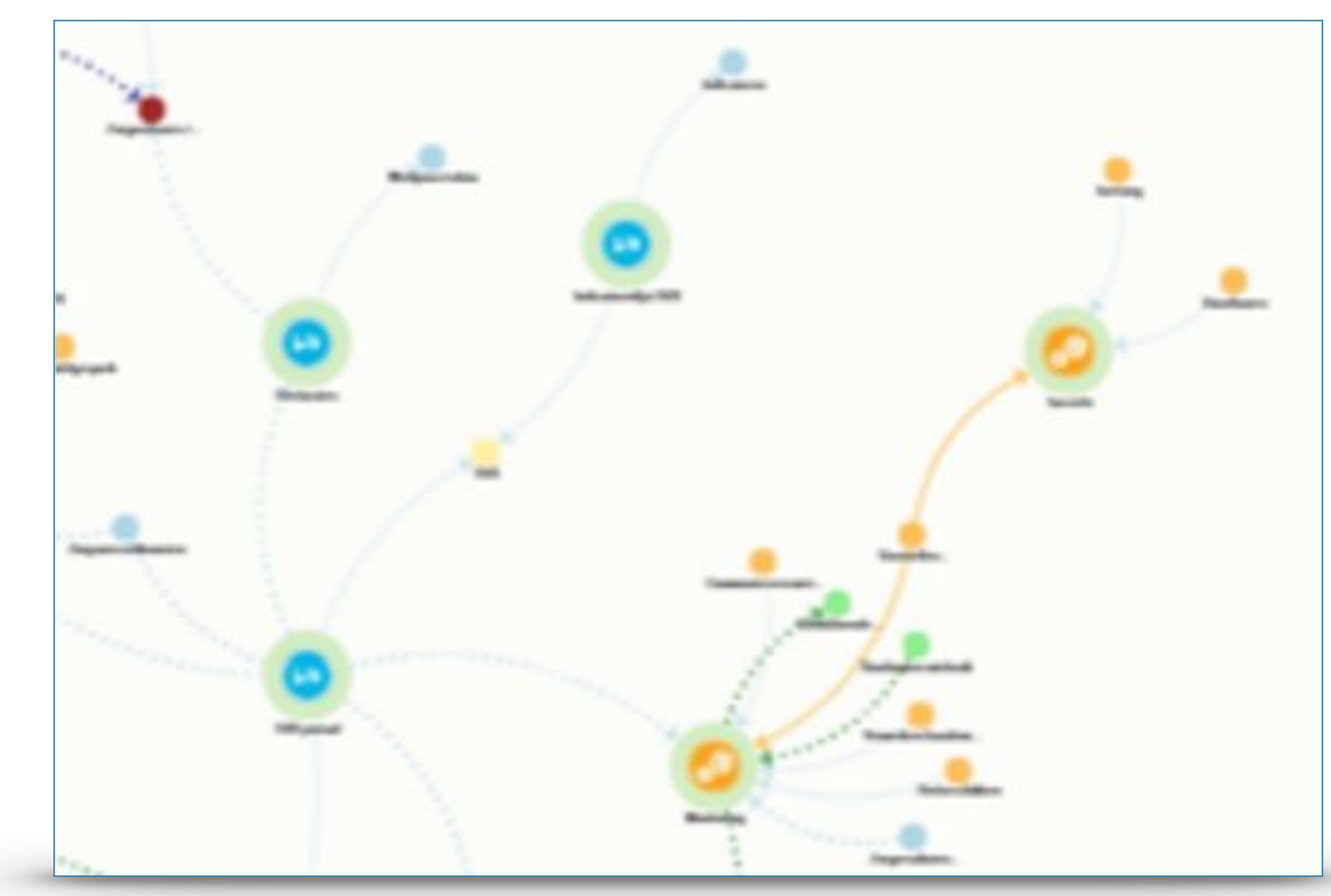

Figure 3: Final force field map (blurred excerpt) produced after seven working sessions

Our emerging methodology has so far focused on supporting the collaborative sensemaking of individual community networks. Of course, it is important that collaborative communities start making sense of themselves through co-creating their own maps and using them in practice, thus developing an active sense of ownership and identity. Still, throughout the process of mapping our case communities, we observed that there are always connections being made to larger stakeholder networks and other communities, such as demonstrated in the above case. To scale for collective impact, sensemaking should therefore extend beyond the individual community network maps. If, as Etzioni (1993) says, society consists of a supra-community, a "community of communities" (or more precisely: a network of social networks and community networks), then the maps embodying their essence should also be linked. How to do this, is still very much an open question. To extend our methodology so that it explicitly supports inter-communal sensemaking, we start with some observations from practice

\section{Making sense across communities: some observations from practice}

Operationally through their networks, but also conceptually through the mapping languages used, there are many interconnections between communities. To explore the nature of such inter-communal sensemaking, we first present four observations that we 
made while involved in actual participatory community network mapping projects: linking maps across communities; meta-communication matters; tweaking the typologies; and sharing community network patterns. We see these observations only as beginnings of better understanding the intricate ways in which separate mapping activities should be embedded in inter-communal network sensemaking and development processes.

\section{Linking maps across communities}

An interesting anecdote from practice shows there may be significant potential to link maps across communities. A local dairy farmer not involved in the Tilburg urban farming community is interested in developing new business models for traditional farms that want to start working more sustainably. Instead of developing yet another "mega-barn", his aim is to reinvent and share farm practices in a process of social innovation, in what he calls an "open source farm lab". Inspired by the Tilburg urban farming community map, the farmer started to develop a stakeholder network map of the community network forming around his own initiative (http://bit.ly/2ocmFKS). The next step is aligning the two maps, examining how they could be used by professional farmers moving towards sustainability to build alliances with urban farmers rooted in the city, thereby strengthening both communities. One supporting role there could be played by public librarians, acting as social innovation catalysts (de Moor, 2015).

\section{Meta-communication matters}

Often, producing the map is seen as the ultimate goal of the community mapping process. However, this initial sensemaking artefact is only a reference point for a community and its surrounding stakeholder network, showing the current or desired state of affairs. By itself, a map does not do much. By presenting the right combinations of elements, connections and perspectives, however, the map may trigger meaningful conversations between community network members and related stakeholders. Especially when trying to build bridges across communities, members from other communities often need to be made aware of the existence of the map through other communication channels than those used by the producing community itself. Generic bridging media such as Twitter are useful for this purpose (Savage, 2011). For example, the master map maker of the Tilburg urban farming community map announced the release of the final version on Twitter. It was not only retweeted by the manager of the overall provincial urban farming communities-project, but also by an unknown urban farmer from a different geographical location, and by the local dairy farmer mentioned above. What (social) media channels (including mailings and newsletters) to use increase cross-community awareness, how to use them effectively, and who should play the linking pin roles is still ill-understood.

\section{Tweaking the typologies}

The basic element and connection typologies developed for the Tilburg urban farming community case were applied in several other early community and network mapping projects, amongst others a university science hub, a regional social innovation network association, a provincial public library association, and two centers of expertise. We observed that the element and connections types were generally reusable, but needed to 
be subtly adapted in different ways in the various cases. For example, core element types in the Tilburg urban farming community case were Participants, Activities, Results, and Tools.

Another initial community network mapping project we were involved in is the Science Hub Brabant. In the Netherlands, there exists a national network of so-called "science hubs", each associated with one or more universities. The mission of these hubs is to find innovative ways to get primary school-aged children interested in science. The core activities of one of these hubs, the Science Hub Brabant include, for instance, 'Kids' Lectures", in which professors give tailor-made lectures about their topics of specialization to groups of children in a university lecture hall; a "Junior Science Café", in which researchers discuss their work with children and perform small experiments with the children being co-researchers; and the "Kids' Knowledge Base", in which digital resources are developed for home and classroom use in order to introduce children to academic fields and themes (de Moor, 2014). However, the Science Hub having limited resources, it leverages its core activities by linking them to activities organized by other organizations. In its community network map, therefore, there is the need to distinguish between Core activities and (secondary) Activities (Figure 4, see for the full current map http://bit.ly/2nqN1ZL).

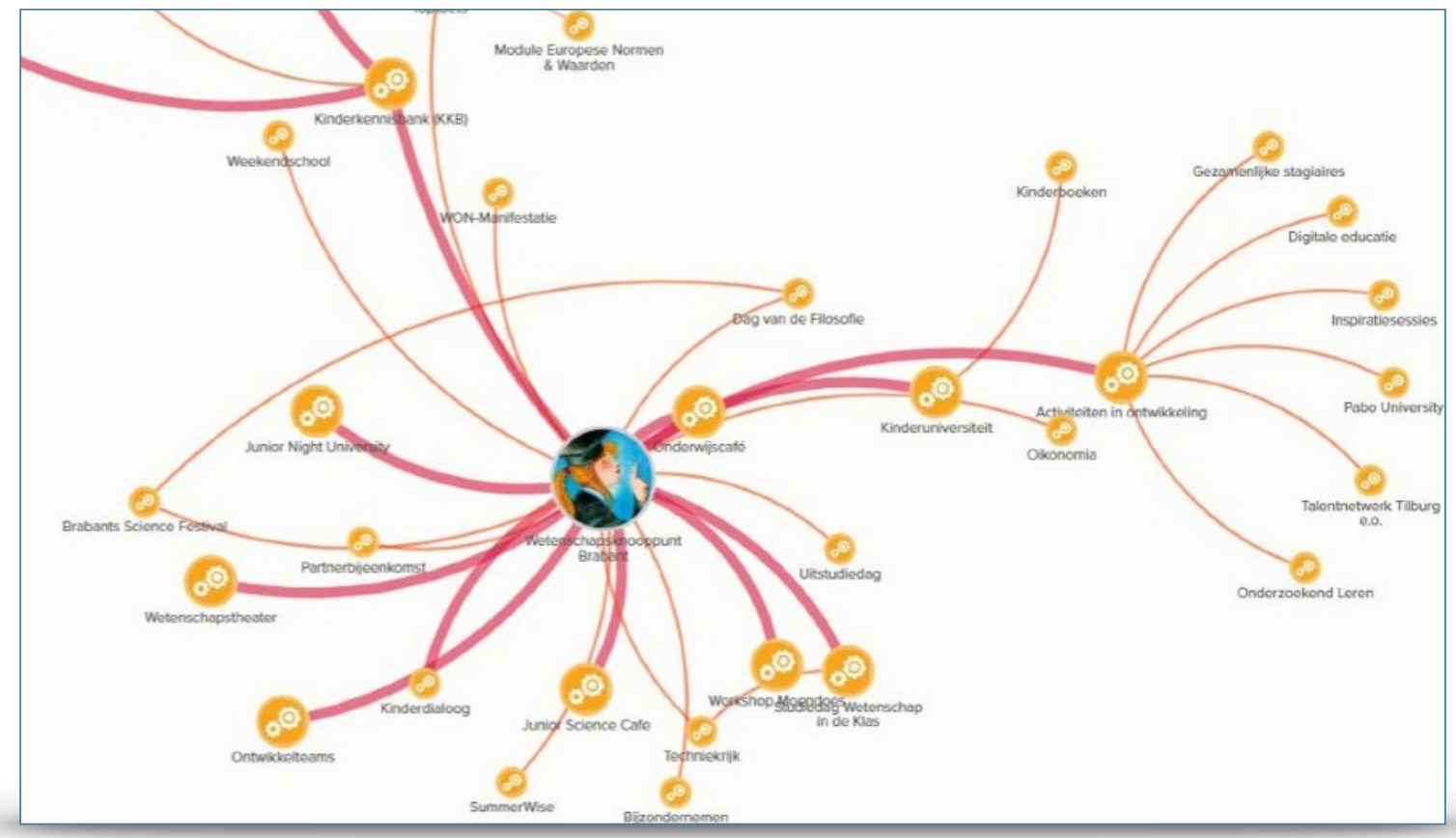

Figure 4: Science Hub Brabant map (excerpt) showing networked primary (large) and secondary (smaller) activities

When communities start collaborating, it is important to make sense of what element and connection types can act as boundary spanners, so that a well-understood collaborative mesh can emerge across the communities. Well-selected, situated types can be important boundary objects. Such objects help broker translation, coordination and alignment among the perspectives of different communities coming together 
(Fischer \& Shipman, 2011). Work on defining and aligning intra and inter-community element and connection typologies is still in its infancy, however.

\section{Sharing community network patterns}

Maps can serve different sensemaking purposes. Community maps at first sight are only descriptive of their own community network case: charting who is relating to and interacting with whom about what. However, we also discovered that (generalized) map fragments can be reused within and across cases. For example, map fragments describing the generic types of roles and content involved in activities in the Tilburg urban farming community case were - with some tweaking - reusable in the map of the Science Hub Brabant. Another example of how multiple communities of practice can be linked through common project and domain-elements is presented in the next section. Such generalized fragments are in fact community collaboration patterns, outlining potential community relationships and interactions relevant to making the community more collaborative. These patterns are not rigid procedures to be followed literally. Instead, they are to be taken as sensemaking starters, to be interpreted and further detailed in an active process of conversation and reflection by community members. Such patterns are therefore not prescriptive, but generative, weakening barriers to and creating opportunities for thinking or acting that promote collective/civic intelligence (Schuler et al., 2011).

When taking a closer look at our inter-communal sensemaking observations, we see they are of two different types:

First of all, inter-communal sensemaking allows multiple communities to build bridges and work together more effectively by aligning their practices at the operational level, together forming a higher order networked communitysystem. Society itself being made of many interconnected communities makes this need clear. In (de Moor, 2015), we showed an example of a collective sensemaking process bridging the interests of a theater community and an environmental cafe community. Patterns are a useful instrument to inform the building of such bridges, as they, for instance, help identify potential social objects to which different communities can jointly relate.

Second, (meta-level) sense needs to be made across communities - sometimes even in different domains - by sharing their generalized lessons learnt. Often, communities find practical solutions for their collaboration problems that could be appropriated and reused in other, unrelated communities. As communities are situated, these solutions cannot be blueprints, but need to be in a form that can fit the specific "collaboration ecosystem" of the receiving community. In other words, lessons learnt need to be abstracted to the right extent, sufficiently specific so that they are still useful, but generic enough to remain usable across often widely different cases. 


\section{Towards pattern-driven participatory community network mapping}

Many community network mapping projects start from conceptual scratch. We believe, however, that distilling, sharing, combining and re-configuring good practice-patterns may be an important step in increasing the efficacy and impact of participatory community network mapping, especially in the context of goal-oriented collaborative communities. To this purpose, we propose the development of a community collaboration pattern language.

\section{Towards a community collaboration pattern language}

Collaboration patterns capture socio-technical lessons learnt in optimizing the effectiveness and efficiency of collaboration processes (de Moor, 2009). In a community setting, these patterns help discover and build the collaborative context in which the community interactions take place (e.g. the goals, roles, content, and tools associated with the interactions).

Pattern languages are networks of patterns that call upon one another. Pattern languages can help promote creativity, collaborative and critical thinking, while acting as a metalanguage that enables people with different roles to communicate and share experiences with each other (Pan \& Stolterman, 2013). In particular for building bridges across communities, pattern languages are useful, as there are often not many existing intercommunal links yet, with community builders from across the communal divide unsure about what could be potential common ground. A particularly good example of an initiative addressing this problem is the Liberating Voices pattern language (Schuler, 2008), with its strong focus on fostering societal empowerment and civic intelligence. Common sense can be made by representatives from various communities following a suggested path between multiple patterns, in the meantime interpreting their joint context through the lens of these patterns. For example, a group of stakeholders could start with interpreting the Collective Decision Making-pattern (http:// publicsphereproject.org/node/209), then together selecting and discussing the most effective implementation of one of the suggested pathways from that pattern, such as the Multi-Party Negotiation for Conflict Resolution-pattern (http:// www.publicsphereproject.org/node/278).

Good pattern languages take time to develop, as they need to transcend particular situations or problems (Pan \& Stolterman, 2013). Developing a pattern language is a form of ongoing, cross-case grounded theory development. Classic grounded theory develops conceptualized theory from the ground up by coding observations, organizing codes, comparing them, selectively coding for identified core variables, and examining the emerging relationships between categories identified (Chametzky, 2016), thus inductively building a conceptual model. It is along similar lines - but absorbing insights from many cases rather than a single in-depth analysis - that community collaboration patterns can be constructed, and further evolve across cases.

We next illustrate how we are constructing such a community collaboration pattern language. We outline the actual emergence of an important class of community collaboration patterns in our community network mapping practice: core community 
interaction patterns - which form the conceptual backbone of the pattern language. Note that for lack of space we leave out many of the details. Important here is to get an overall sense of the evolutionary process.

\section{The cross-case evolution of core community interaction patterns}

At the heart of collaboration patterns are community interactions. In this section, we show how a core community interaction pattern evolved across several cases.

\section{The initial Core Community Interaction Pattern}

Our quest for identifying collaboration patterns started by framing an initial core interaction pattern grounded in earlier work on socio-technical community collaboration patterns (de Moor, 2009) and Carrol and Rosson's conceptual model of community (in Carroll, 2012, p.15). In this paper, we will use a simplified version, outlining only the core conceptual elements and connections of this pattern (Figure 5).

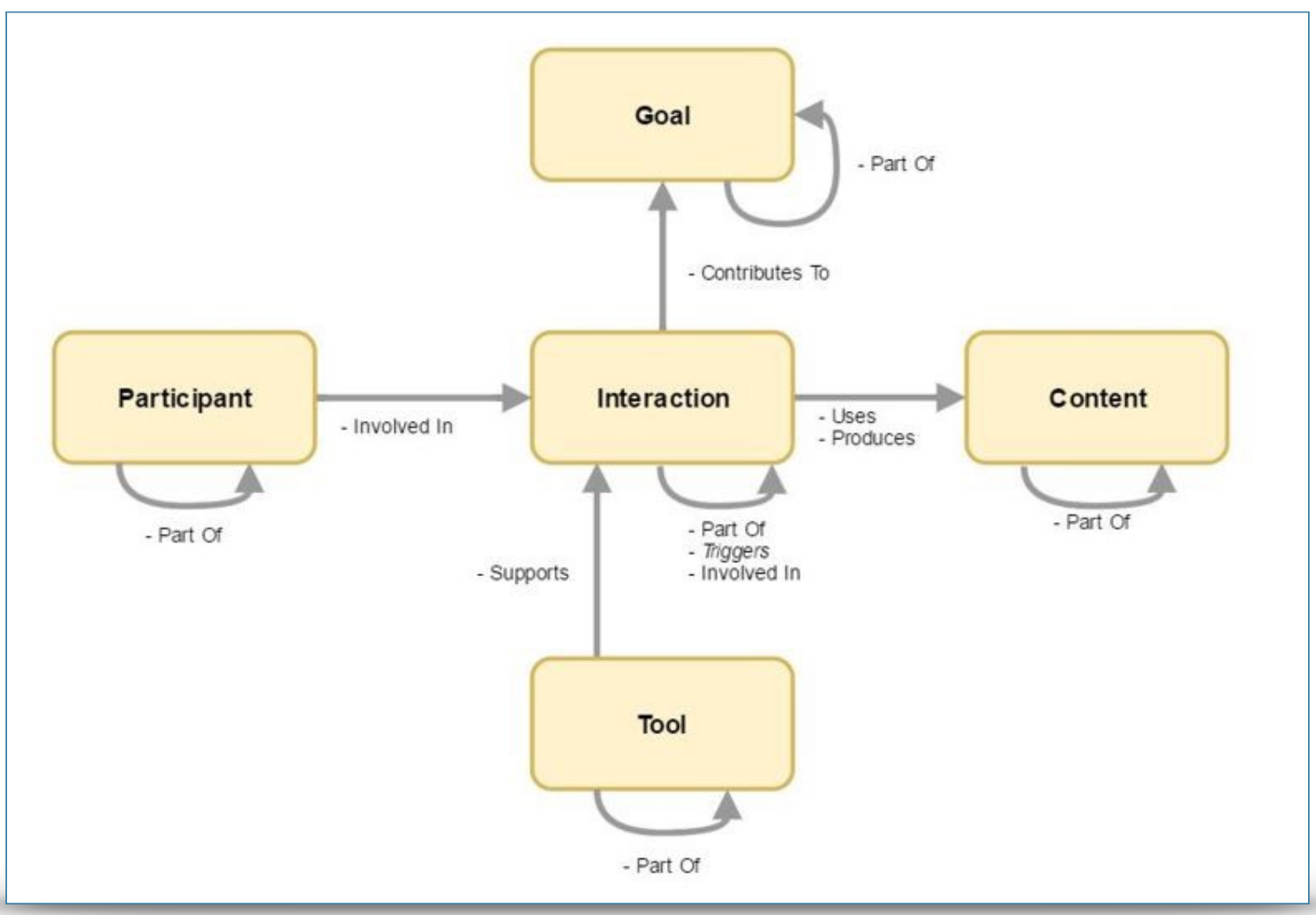

Figure 5: The initial Core Community Interaction Pattern

What this community network building block says, is that each Interaction may Contribute To Goals, may be Part Of, Trigger, or be Involved in other Interactions, Use or Produce various types of Content, Involve various types of Participants, and be Supported by — sometimes a whole ecosystem of — physical and online Tools. 
Furthermore, all of these elements can be Part Of other elements of the same type (e.g. . an Organization Department can be Part Of an Organization)3.

Figures 6 and 7 shows two adaptations of this basic pattern in subsequent cases. They were decided upon after in-depth discussions about map language, tools, and processes and presentations of the draft maps to community managers and selected core community members, going through various iterations. We take their thinking these patterns to be understandable and useful to be a promising measure for the validity of these patterns, tentative as they are.

\section{The Tilburg Urban Farming Community case}

Figure 6 (see next page) shows an initial adaptation of the core community interaction pattern driving the mapping process in the Tilburg Urban Farming Community case. We see that key Interactions in this community were called Activities. Three different types of Participants were distinguished: Individuals, Organizations, and Communities. The type of Content of special interest to this community were Results Produced in the Activities (Used Content was not visualized as it was not essential for the current sensemaking effort to understand how the activities were organized). As the Results were official project deliverables, they also acted as Goals. In addition to being Involved, Participants could be related to Activities by just being kept Informed about them. Note that, according to the initial core community interaction pattern, Results can be Part Of other Results. However, modeling details these was not of interest to this community, at least in this stage of its development, as the main focus was on first outlining the overall network of Activities. Note that the element and connection types of the generic core community interaction pattern that were created for this case are indicated in red and those not used in this case are represented in italics. For example, Contributes To and Produces were not used in the mapping discussions, as their relation subtype in common - Has Result - was more relevant to the (initial) sensemaking purpose of this community.

\section{The RDM Center of Expertise case}

Figure 7 (see following page) is another variation of the initial core community interaction pattern. It was at the heart of a mapping exercise to support community network building in the RDM Centre of Expertise $(\mathrm{CoE})$ in Rotterdam, coordinated by the Rotterdam University of Applied Sciences. The CoE has as its mission to develop better technical education, as well as new knowledge and sustainable innovations required by the Port and City of Rotterdam. It does so by supporting collaboration between educational institutes, research centers and corporations in a range of projects, also involving university lecturers and students. This collaboration takes place in a network of currently seven communities of practice (CoPs).

3 A good example of meaning evolution across patterns is that Part Of in later cases turned out to also often be naturally used to connect elements that are in some broad sense part of elements of different types, for example a specific tool or data source being maintained ("part of") an organization. How exactly to deal with this difference between actual informal use and intended formal interpretation is a topic of ongoing investigation. 


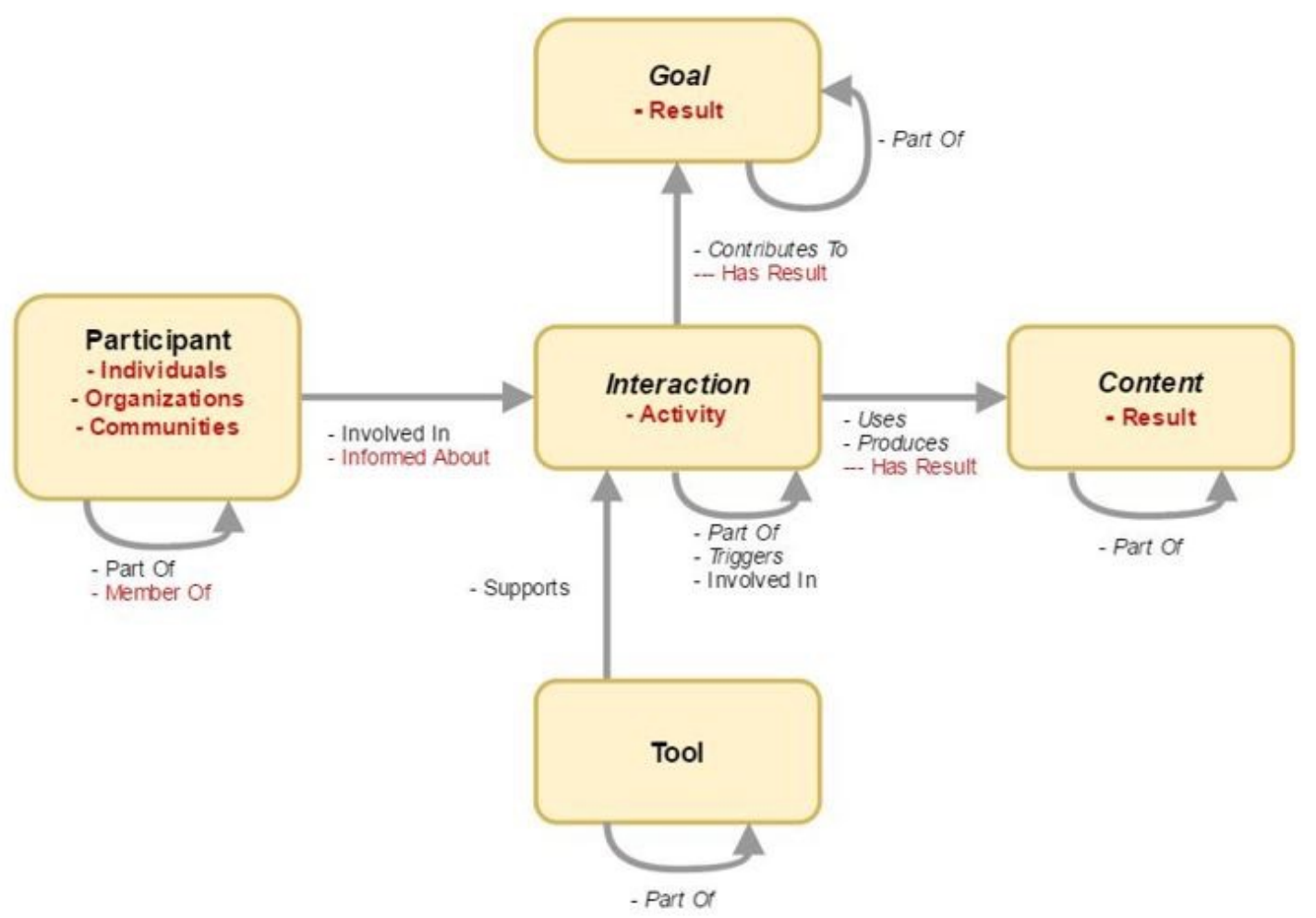

Figure 6: The Core Tilburg Urban Farming Community Interaction Pattern

Community network mapping was considered to have potential to visualize the collaboration ecosystem not only within but especially across the various communities. To explore this potential, a pilot was conducted with two of the communities of practice (CoPs): the CoP Logistics and the CoP Future Mobility. These communities were selected as the community managers were already exploring cross-overs between the projects associated with their communities. In several iterations, a pilot map was produced (https://kumu.io/rdm-coe/rdm-coe). This map is now being extended by the community managers and researchers of the $\mathrm{CoE}$ to make it cover increasingly more common ground.

The basic unit of Interaction in the CoE are Projects. An auxiliary Interaction concerns Educational Activity, which itself is associated with a special type of Participant: Educational Program (as an organizational structure). This relationship is considered essential for successful operations of the $\mathrm{CoE}$, as much of the research capacity comes from university lecturers and students. Having this relationship modeled in the map will make discovery of relevant Projects by, say a student enrolled in a particular Educational Activity much easier. A key Goal context of those Projects are one of four Domains the CoE is working on. Key Participants to be modeled are - next to the Communities of Practice the Organizations involved as participants, sponsors or owners of the Projects. Representing selected individual persons can be relevant, for example, as project contacts, but are modeled as attributes of project elements instead of as separate map elements. This to keep the maps maintainable and focused on the (project) essence of the collaboration structure of the CoE. Two special types of relation between 
Projects and Participants are Sponsors and Owners, as this is key resource information for further network building. No Tools and Content were modeled, as - so far - the map is especially used for communicating the overall collaboration network structure, not to zoom in on the project level.

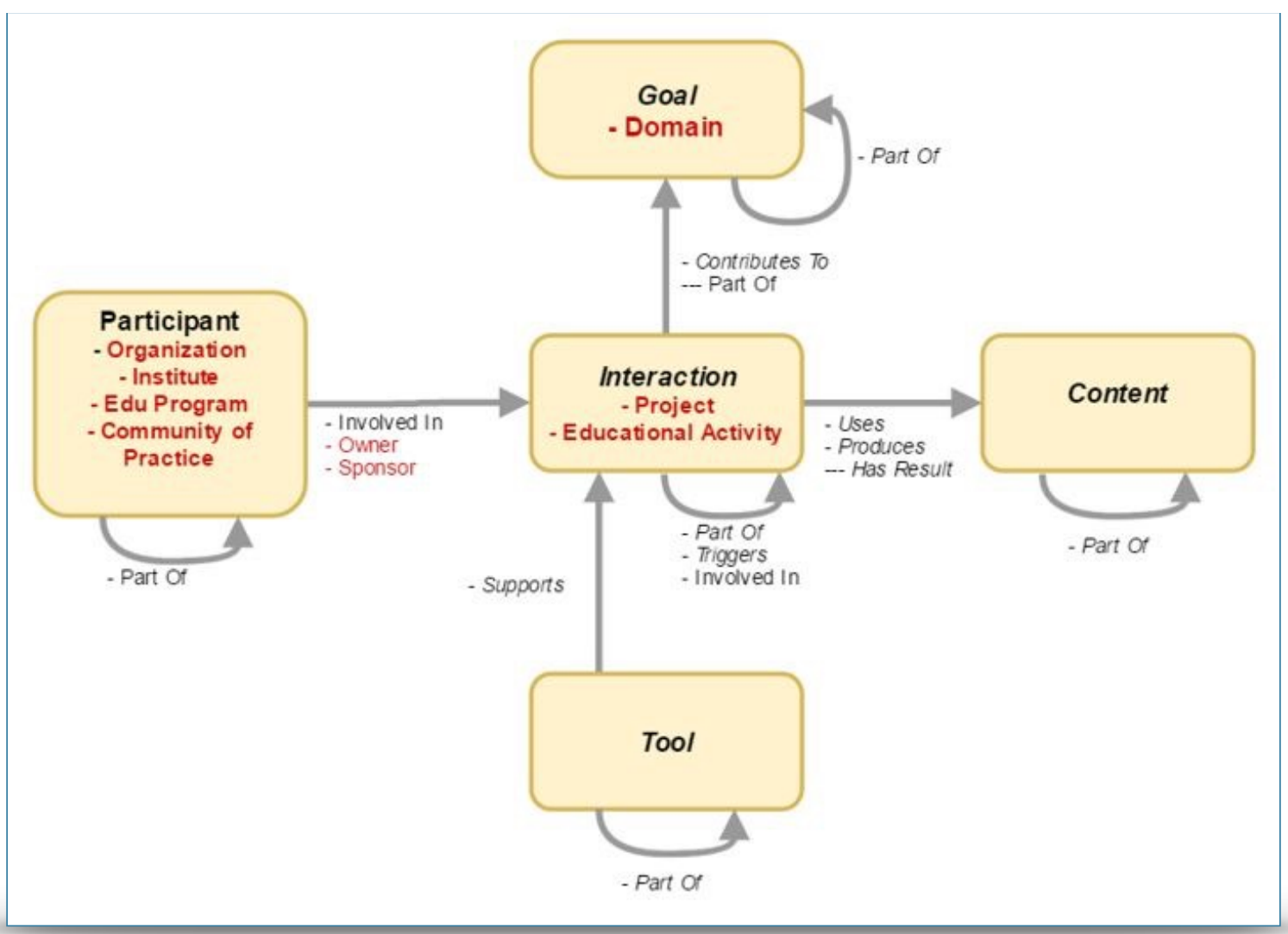

Figure 7: The Core RDM Centre of Expertise Community Interaction Pattern

Figure 8 (see next page) shows an implementation of this pattern in the RDM CoE pilot map. It is a submap that zooms in on one of the projects, INTRALOG. The meaning of the various symbols is indicated next to the map. The red lines on the map indicate involvement-relations, the blue ones membership-relations, the dashed orange and brown lines ownership and sponsorship relations, respectively. 


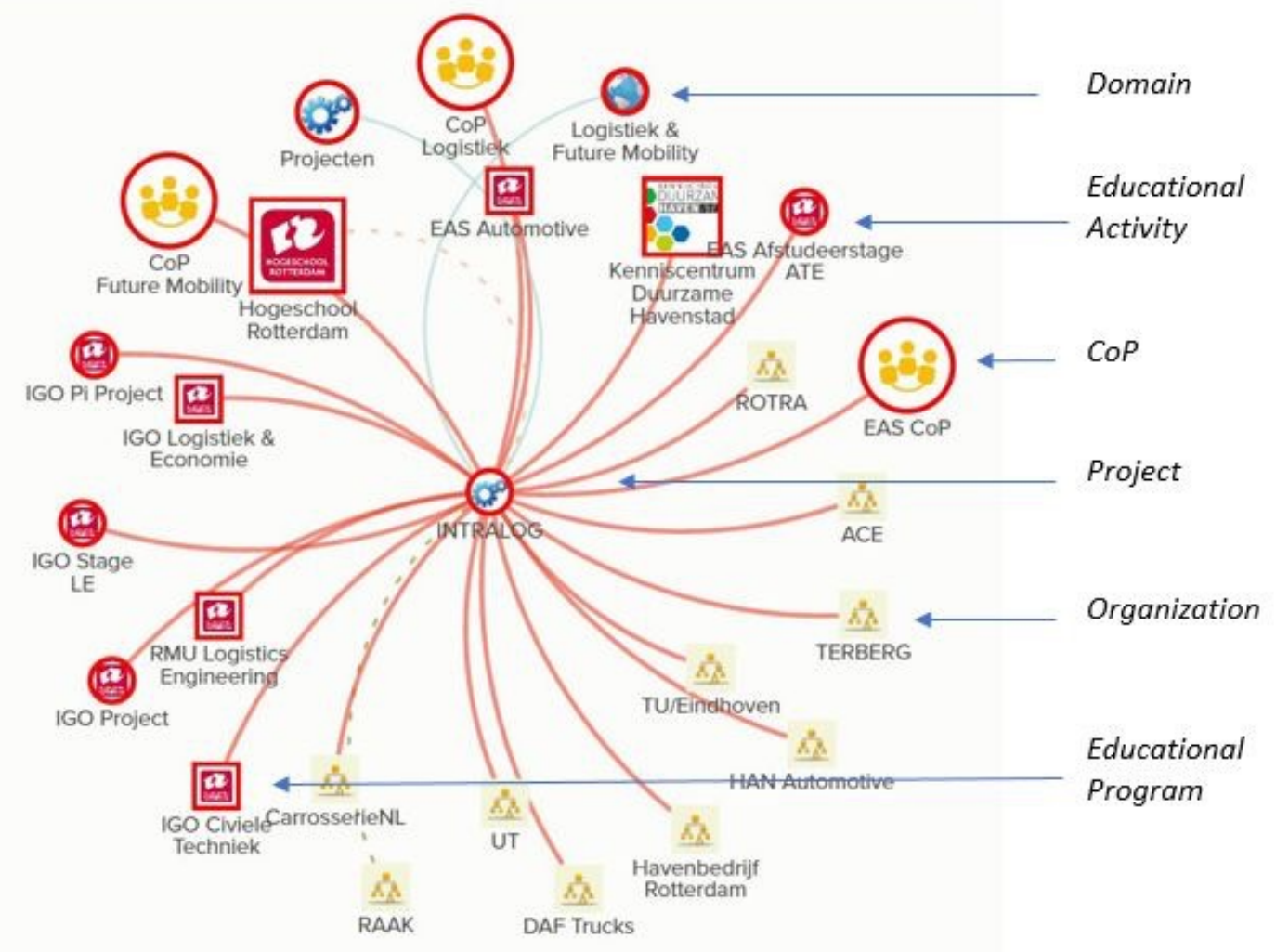

Figure 8: Implementation of the Core RDM Centre of Expertise Community Interaction Pattern in the INTRALOG project sub map

\section{Discussion}

In this article, we explained how we are developing CommunitySensor, a participatory community network mapping methodology. We started by summing up the current state of the methodology. It essentially consists of two interlocking mapping-driven cycles: the Community Network Development Cycle, comprised of a community network mapping, sensemaking, building, and evaluation stage. We then zoomed in on the heart of the methodology - the Community Network Sensemaking Cycle - in which typically the network mapping and sensemaking stages are repeated a number of times in order to arrive at the issues, priorities, and next actions that inform the next set of interventions in developing the community network.

Most of our methodology development attention has so far been paid to the mapping and sensemaking activities. However, we also plan to more systematically work on the subsequent community building interventions that actually make the community network grow, and the evaluation activities that provide the inputs for the next round of community mapping efforts. For expanding these subsequent community network building and evaluation stages, we build on existing practices from (Wenger et al, 2002; Wenger et al., 2011). Combining such participatory mapping-powered communitybuilding and evaluation processes with scalable collaboration platform development 
processes (White et al., 2014), could further increase the collective impact of community network development activities.

The participatory nature of the community network mapping process plays out in several ways. First of all, community representatives are strongly involved in defining the language to be used, in particular what types of elements and connections are most relevant to their community. They also define what perspectives on the map matter to them, so that attention of their members is best directed to what matters most in literally - their view. In terms of process participation, data for the maps is largely provided by the community members themselves, for example in the form of surveys, spreadsheets, and interviews. Especially the level of participation in the maintenance and use of the maps can still be improved. Right now, the consultant, as "master map maker" is designing the architecture and making the seed map. Community members have been trained in several instances to do basic maintenance (adding new elements and connections of the same type), but more complex map maintenance tasks such as changing the structure and layout of the map and adding new features is still too complex for most. Furthermore, how to put maps to sustained community use, for example by weaving them into regular community sensemaking activities, such as community meetings, and integrating them into primary processes (such as research and education in the RDM case) needs more thought.

Stimulating engaging physical stakeholder conversations about the maps and their generating patterns seems essential to engender community ownership and adoption. We have conducted experiments with different Sensemaking Cycle formats, of which the force field analysis case is a typical example. However, we have also tried very different formats, such as having participants talk to each other one-on-one, then letting them tag each other with concepts they think best describe their conversation partner professionally, immediately adding those concepts and their connections to the map, followed by plenary group discussion of what the group has in common as the basis for further action (http://bit.ly/2tunIHC). We are still making sense of the many conceptual links between mapping and sensemaking, as this is such a rich field of inquiry. To further our understanding, we aim to build on existing related sensemaking work, such as group facilitation, e.g. (Justice \& Jamieson, 2006), network weaving (Holley, 2013), dialogue mapping (Conklin, 2006), and participatory representation practice, which focuses on the interplay between facilitators and participants, specifically how practitioners make participatory visual representations coherent, engaging, and useful (Selvin, 2011).

So far, we have focused on charting the existing "ecosystems" of participants and stakeholders. Still, visualizing what "is" is not enough. Equally important is to model the intentions and aspirations, the goals, of the evolving community network. One important $\mathrm{R} \& \mathrm{D}$ focus is how to bring goals more explicitly into the equation. We dub this "visualizing the GAP", the many kinds of instantiations of and relationships between Goals-Activities-Participants. We already found that modeling "themes" is a very useful bridge to move from the existing ("is") to the aspirational ("ought"). Themes are open-ended and inspire generative conversation, often more so than existing official project objectives, for example, with their frequently constraining connotations 
like office politics and budgets. There is a wide range of models we can draw from to develop this intentional dimension, such as the social innovation process and network models provided by the literature (Murray \& Mulgan, 2010; de Moor, 2013).

Besides extending the intentional/aspirational in our mapping methodology, we also need to address the issue of how to use mapping to evaluate the impact of the community networks. For collective impact, the relational is as important as the rational, and structure is as important as strategy (Kania et al., 2014). One direction to explore is how social capital evaluation frameworks such as by Marais (2012) can inform the analysis of what connections in maps matter. Social network analysis provides us with basic measures to capture essential structural properties of social networks, such as degree centrality, closeness and betweenness. Such measures are visualized by the Kumu network visualization tool (http://kumu.io) we use in our community mapping projects. In participatory mapping sessions, this social network analysis feature allows the facilitator to, for example, on the fly identify potential hubs and "movers and shakers" in the community, the interpretation of which is then to be discussed with the community representatives present. Other Kumu tool features include visually indicating impact, for example, using layout to show weighted metrics (e.g. larger elements depending on the size of one of its attributes or the number/weight of connections it has). We intend to experiment with these features in upcoming mapping projects. Developing sensible visualizations and mapping processes in which to create and interpret them can help prevent an overly quantitative approach to measuring community value, which runs the risk of not capturing the essence of what the community is really about (Wenger et al., 2011).

Still, a foundation of our methodology construction project is not just about looking inward - at the particular community network at hand - but also to see how it can draw from - and contribute to a larger commons. We believe this to be essential in overcoming fragmentation and increasing collective impact. We have therefore examined the role that participatory community network mapping can play in making sense not only within, but also across communities. We identified first order intercommunal sensemaking, where actual connections are made between different communities in a particular case, and second-order inter-communal sensemaking, in which generalized lessons learnt are drawn and shared across cases.

Patterns help improve both types of inter-communal sensemaking. We described how we are bootstrapping a pattern-driven methodology, with the ultimate aim of developing a community collaboration pattern language that can seed and catalyze this process. We illustrated the role patterns play by examining the evolution of core community interaction patterns across several cases. The patterns are only tentative and need further specification to meet formal knowledge representation standards. Still, they show proof of concept of how such patterns can adapt and become re-usable, forming the "collaboration genome" of a larger society as it were. Although both mapping methodology and collaboration pattern language are still in an early stage, they continue to develop rapidly. 
Ontologies are explicit (often formal) specifications of conceptualizations (Gruber, 1994), necessary if patterns are to defined consistently and in a reusable and scalable way. There are many ways to represent ontologies. In (de Moor, 2009; de Moor, 2013), for instance, we show how the ontologies underlying our collaboration patterns can be represented using the Conceptual Graphs formalism. However, formal knowledge representations are not enough, there needs to be a process to apply, interpret, and evolve the pattern representations. One stream of relevant work we draw from is collaborative ontology engineering. This approach sees ontology engineering as a consensus-building process in which stakeholders/communities of practice agree upon a common view of a domain of interests, their shared knowledge being structured in terms of concepts, attributes, relationships and constraints (Simperl \& Luczak-Rösch, 2014). This stream of research has developed many formal approaches to conceptual model distillation, alignment, and conflict resolution which could help us validate and organize the proto-patterns emerging from cases as discussed in the previous section. Vice versa, our work could inform this field in terms of the interplay between real-world community sensemaking and the often very abstract knowledge representations that make up formal ontologies.

Another input for pattern language development are social network analysis approaches, for which the Kumu tool already provides some basic features, as mentioned earlier. For example, by analyzing social media conversation networks, community clusters can be discovered (Smith et al., 2014). Structural social network analysis and ontology engineering approaches merge in new research streams like semantic mining of social networks (Tang \& Li, 2015), which could further help to expand pattern language construction.

A weakness of many pattern languages is that most of their efforts seem to go into creating the pattern language, rather than using it, leading to insufficient analysis and evaluation of pattern languages in action (Pan and Stolterman, 2013). To develop an effective pattern-driven participatory mapping methodology, creating, analyzing, and comparing pattern use cases is paramount. Through our approach of reflecting upon multiple hands-on mapping projects, examining what patterns are being used and can be distilled in each case, while simultaneously developing a reusable collection of community collaboration patterns, we hope to contribute to this still emerging body of knowledge. To further enrich these inter-active aspects of our methodology, we are inspired by related community-focused pattern language work, and the meta-design of socio-technical systems. For example, the Liberating Voices pattern language has developed a range of pattern-driven workshops and games (Schuler, 2011). Work on the meta-design of socio-technical systems provides us with more general process-oriented principles on which to build our methodology, such as cultures of participation, empowerment for adaptation and evolution, and seeding and evolutionary growth (Fischer \& Herrmann, 2011).

\section{Conclusion}

To achieve global collective impact, we need to considerably grow and put to much better use civic intelligence and social innovation capacity. Top-down approaches led by 
governments and large corporations are insufficient. Networked communities are a core part of the societal fabric required. We need more systematic approaches for community networks to examine and improve their intra- and inter-communal collaboration, to which participatory community network mapping is instrumental. We outlined our emerging methodology for supporting this process: CommunitySensor. However, supporting individual community mapping projects from scratch and in isolation is not enough. We argue that collaboration patterns are key in scaling up sensemaking and community network development processes towards collective impact: they form an evolving ecosystem of units of collaborative meaning and lessons learnt that can help seed, link, and strengthen community network development and collaboration. Pattern-driven participatory community network mapping should be a constitutive process for making, growing, and applying the inter-communal sensemaking capacity essential to achieve sustainable global change. We hope that the emerging methodology outlined in this paper provides some of the theoretical and practical scaffolding on which to build future participatory community network mapping R\&D, implementation, adoption and use efforts.

\section{Acknowledgements}

This article is a revised and extended version of the paper "Collaborative Sensemaking: Bootstrapping a Pattern-Driven Participatory Community Mapping Methodology" which was accepted for publication and presented at the 13th Prato CIRN Conference 2-4 November 2016, Monash Centre, Prato Italy.

\section{References}

Andrews, D. (2002). Audience-Specific Online Community Design. Communications of the $A C M, 45(4), 64-68$.

Carroll, J. M. (2012). The Neighborhood in the Internet: Design Research Projects in Community Informatics (1st ed.). New York, NY: Routledge.

Chametzky, B. (2016). Coding in Classic Grounded Theory: I've Done an Interview; Now What? Sociology Mind, 6(4), 163-172.

Conklin, J. (2006). Wicked Problems and Social Complexity. In Dialog Mapping: Building Shared Understanding of Wicked Problems (pp. 3-40). Hoboken, N.J: John Wiley \& Sons.R. E. Anderson.

de Moor, A. (2009). Collaboration Patterns as Building Blocks for Community Informatics. In Proc. of the 6th Prato Community Informatics Research Network Conference. Prato, Italy.

de Moor, A. (2013). Creativity Meets Rationale: Collaboration Patterns for Social Innovation. In J. Carroll (Ed.), Creativity and Rationale: Enhancing Human Experience by Design (pp. 377-404). Springer.

de Moor, A. (2014). The "Kids' Knowledge Base": Connecting Junior Science to Society. In Proc. of Chi Sparks 2014 "Creating the Difference", The Hague, the Netherlands, pp. 108-111. 
de Moor, A. (2015). Knowledge Weaving for Social Innovation: Laying the First Strand. In Proc. of the 12th Prato Community Informatics Research Network Conference, Prato, Italy.

de Moor, A. (2015b). Towards a Participatory Community Mapping Method: The Tilburg Urban Farming Community Case. In G. Avram, F. De Cindio, \& V. Pipek (Eds.), Proc. of the Work-In-Progress Track of the 7th International Conference on Communities and Technologies, Limerick, Ireland, 27-30 June, 2015.

Etzioni, A. (1993). The Spirit of Community: The Reinvention of American Society. New York: Simon \& Schuster.

Fischer, G., \& Shipman, F. (2011). Collaborative Design Rationale and Social Creativity in Cultures of Participation. Human Technology, 7(2), 164-187.

Fischer, G., \& Herrmann, T. (2011). Socio-Technical Systems: A Meta-Design Perspective. International Journal of Sociotechnology and Knowledge Development (IJSKD), 3(1), $1-33$.

Gruber, T. (1994). Towards Principles for the Design of Ontologies Used for Knowledge Sharing. In Formal Ontology in Conceptual Analysis and Knowledge Representation. Kluwer.

Holley, J. (2013). Introduction to Network Weaving. Athens, Ohio: Network Weaver Publishing.

Irby, M., \& Boyle, P. (2014). Aligning Collective Impact Initiatives. Stanford Social Innovation Review, (Collective Insights on Collective Impact), Fall 2014, 15-16.

Justice, T., \& Jamieson, D. W. (2006). The Facilitator's Fieldbook (2nd edition). New York: AMACOM.

Kania, J., Hanleybrown, F., \& Splansky Juster, J. (2014). Essential Mindset Shifts for Collective Impact. Stanford Social Innovation Review, (Collective Insights on Collective Impact), Fall 2014, 2-5.

Klein, N. (2015). This Changes Everything: Capitalism Vs. The Climate. New York, NY: Penguin Books.

Marais, M. A. (2012). An Overview of the Role of Social Capital in Development Processes. In Proc. of CIRN 2012 Community Informatics Conference: Ideals meet Reality. Prato, Italy.

Murray, R., Caulier-Grice, J., \& Mulgan, G. (2010). The Open Book of Social Innovation. NESTA \& Young Foundation. Retrieved from http://www.nesta.org.uk/publications/ assets/features/the open_book of social_innovation

Novak, J. D., \& Cañas, A. J. (2008). The Theory Underlying Concept Maps and How to Construct and Use Them (Technical Report IHMC CmapTools No. 2006-1-2008). Institute for Human and Machine Cognition.

Pan, Y., \& Stolterman, E. (2013, April). Pattern language and HCI: expectations and experiences. In CHI'13 Extended Abstracts on Human Factors in Computing Systems (pp. 1989-1998). ACM.

Savage, N. (2011). Twitter as Medium and Message. Commun. ACM, 54(3), 18-20

Schuler, D. (2008). Liberating Voices: A Pattern Language for Communication Revolution. Cambridge, Mass.: MIT Press.

Schuler, D., Gillgren, K., \& O’Neil, M. (2011). Pattern Workshops and Pattern Games Generating Civic Intelligence with the Liberating Voices Pattern Language. In 2011 
PUARL International Conference, "Generative Processes, Patterns and the Urban Challenge.

Selvin, A. (2011). Making Representations Matter: Understanding Practitioner Experience in Participatory Sensemaking (PhD thesis). The Open University, UK.

Selvin, A., \& Shum, S. B. (2014). Constructing Knowledge Art: An Experiential Perspective on Crafting Participatory Representations. (J. M. Carroll, Ed.) (Vol. 7). Morgan \& Claypool.

Simperl, E., \& Luczak-Rösch, M. (2014). Collaborative Ontology Engineering: A Survey. The Knowledge Engineering Review, 29(1), 101-131.

Smith, M. (2014). NodeXL: Simple Network Analysis for Social Media. In Encyclopedia of Social Network Analysis and Mining (pp. 1153-1170). New York, NY: Springer.

Smith, M. A., Rainie, L., Himelboim, I., \& Shneiderman, B. (2014). Mapping Twitter Topic Networks: From Polarized Crowds to Community Clusters. Washington, D.C.: Pew Research Center.

Tang, J., \& Li, J. (2015). Semantic Mining of Social Networks. Synthesis Lectures on the Semantic Web: Theory and Technology, 5(2), 1-205.

Weick, K. (1995). Sensemaking in Organizations (Vol. 3). London: Sage.

Wenger, E., McDermott, R. A., \& Snyder, W. (2002). Cultivating Communities of Practice: A Guide to Managing Knowledge. Boston, Mass: Harvard Business School Press.

Wenger, E., Trayner, B., \& de Laat, M. (2011). Promoting and Assessing Value Creation in Communities and Networks: A Conceptual Framework. the Netherlands: Ruud de Moor Centrum, Open University.

White, N., Cardone, R., \& de Moor, A. (2014). Learning 3.0: Collaborating for Impact in Large Development Organizations. Knowledge Management for Development Journal, 10(3): 21-37 